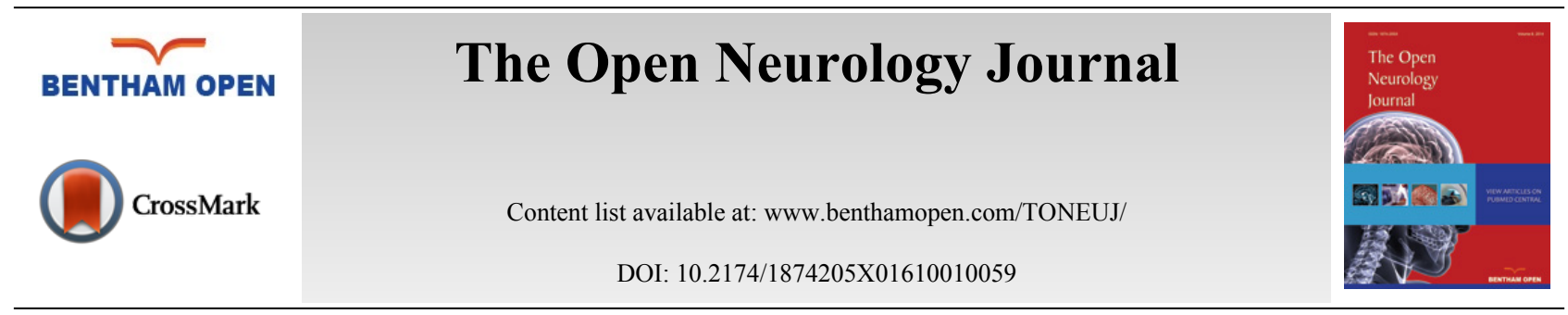

RESEARCH ARTICLE

\title{
Osteopathic Manual Treatment for Amyotrophic Lateral Sclerosis: A Feasibility Pilot Study
}

Alberto Maggiani ${ }^{1, *}$, Lucio Tremolizzo ${ }^{1,2,3,4}$, Andrea Della Valentina ${ }^{1}$, Laurent Mapelli ${ }^{1}$, Silvia Sosio $^{1}$, Valeria Milano ${ }^{1}$, Manuel Bianchi ${ }^{1}$, Francesco Badi ${ }^{1}$, Carolina Lavazza ${ }^{1}$, Marco Grandini ${ }^{1}$, Giovanni Corna ${ }^{4}$, Paola Prometti ${ }^{4}$, Christian Lunetta ${ }^{5}$, Nilo Riva ${ }^{6}$, Alessandra Ferri ${ }^{7}$, Francesca Lanfranconi $^{4}$ and for the ME\&SLA Study ${ }^{\#}$

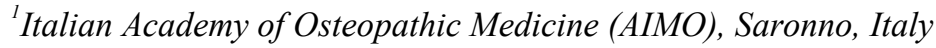

${ }^{2}$ Neurology Unit, "San Gerardo" Hospital, Monza, Italy

${ }^{3}$ Milan-Center for Neuroscience (NeuroMI), Milan, Italy

${ }^{4}$ School of Medicine and Surgery, University of Milano-Bicocca, Monza, Italy

${ }^{5}$ NEuroMuscular Omnicentre (NEMO), Fondazione Serena Onlus, Milano, Italy

"San Raffaele" Scientific Institute, Milano, Italy

${ }^{7}$ Institute of Sport, Exercise and Active Living, Victoria University, Melbourne, Australia

Received: January 6, 2016

Revised: June 18, 2016

Accepted: June 24, 2016

\section{Abstract:}

Background:

Current interventions in amyotrophic lateral sclerosis (ALS) are focused on supporting quality of life (QoL) and easing pain with a multidisciplinary approach.

\section{Objective:}

Primary aim of this pilot work assessed feasibility, safety, tolerability and satisfaction of osteopathic manual treatment (OMT) in 14 ALS outpatients.

\section{Methods:}

Patients were randomized according to an initial single-blind design (12 weeks, T0-T1), in order to receive OMT (weekly for 4 weeks, and fortnightly for the following 8 weeks) versus usual-care ( $\mathrm{n}=7$ each group), followed by an OMT open period (T1-T2, once a week for 8 weeks, $\mathrm{n}=10$ ). Secondary aims included blind osteopathic assessment of somatic dysfunctions (SD) for goal attainment scale (GAS) calculation, Brief Pain Inventory-short form and McGill QoL-16 items.

\section{Results:}

OMT was demonstrated feasible and safe and patients displayed high satisfaction $(\mathrm{T} 1-\mathrm{VAS}=8.34 \pm 0.46 ; \mathrm{T} 2-\mathrm{VAS}=8.52 \pm 0.60)$. Considering secondary aims no significant differences emerged. Finally, at study entry (T0), a cervico-dorsal SD was found in $78 \%$ of ALS patients versus $28 \%$ of healthy matched controls $(p<0.01)$.

\footnotetext{
* Address correspondence to this author at the AIMO, Pizza Santuario 7, 21047 Saronno (VA), Italy; Tel: +39-0296705292; Email: maggiani@aimoosteopatia.qld.it, info@ambulatorioslamonza.it

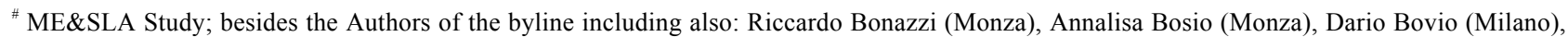
Carlo Ferrarese (Monza), Andrea Magnoni (Monza), Vittorio Mantero (Lecco), Samuele Marchese (Saronno), Ornella Mauri (Monza), Luca Pollastri (Monza), Andrea Rigamonti (Lecco), Caterina Salito (Milano), Vincenzo Silani (Milano), Dino Tenderini (Monza), Barbara Uva (Milano).
} 


\section{Conclusion:}

OMT was found feasible, safe and satisfactory in ALS. The lack of secondary aim differences can be due to the limited sample size. OMT could be an interesting option to explore in ALS.

Keywords: Amyotrophic lateral sclerosis, Feasibility, Osteopathic manual treatment, Pilot trial.

\section{INTRODUCTION}

Amyotrophic lateral sclerosis (ALS) is a neurodegenerative disorder characterized by progressive lack of strength, muscle atrophy and spasticity. The disease usually leads to death in few months since symptom onset due to aspiration pneumonia or respiratory dysfunction. Effective therapies for ALS are currently lacking and management guidelines emphasize the role of a multidisciplinary approach in order to support an adequate quality of life (QoL); emphasis is given on starting cares as soon as possible, providing them until the very end of life. Furthermore, ALS patients often report pain that might easily go underrecognized and undertreated [1]. Among therapies used to face the progressive lack of function in patients with ALS, kinesitherapy, is used in order to avoid excessive spasticity and deconditioning that could overlap with the effects of the disease itself [2]. In this context, osteopathic manual treatment (OMT) might be considered as a complementary approach to ALS, aiming to ameliorate pain management and QoL, as already shown in other neurological disorders, such as Parkinson's disease and headache [3, 4]. Although, to our best knowledge, no literature report is available on OMT in ALS, there is pilot evidence of safety and efficacy of OMT in Parkinson's disease (PD) [3, 5] Understanding the specific impaired or altered function of the somatic system (somatic dysfunctions, SD [6]) expressed by ALS patients might be useful for creating the basis for an effective OMT approach. Finally, OMT is usually well tolerated with a low rate of side effects [4], albeit this has never been reported before in vulnerable patients such as those affected by ALS.

The primary aim of this work consisted in assessing safety, feasibility, tolerability and satisfaction of OMT in a preliminary series of ALS outpatients. The secondary aims included the blind assessment of pain and QoL, besides the blind evaluation of the effects of the OMT by goal attainment scaling (GAS).

\section{MATERIALS AND METHODOLOGY}

\section{Patients}

Following approval of the protocol by the ethics committee, 14 consecutive ALS outpatients were recruited with written informed consent. All clinical investigation were conducted according to the principles expressed in the Declaration of Helsinki.

Inclusion criteria were: diagnosis of possible-, probable- or definite-ALS according to El Escorial criteria [7]; ALS functional rating scale (ALSFRS-R) score $>24$; pulse oximetry values $\geq 92 \%$ in clinostatism. Exclusion criteria were: presence of non-invasive ventilation (NIV) or tracheostomy; clinically relevant orthopnea or pulse oximetry values $<92 \%$ in clinostatism.

Clinical characterization of ALS included the ALSFRS-R [8], the ALS-severity scale (ALS-SS [9],) and the Norris ALS scale [10]. The disease progression index (DPI) was estimated in this work as the loss of ALSFRS-R score over time since onset: [48 - current ALSFRS-R]/disease duration in months [11]. Finally, 14 age- ( \pm 5 years) and sexmatched healthy controls were also recruited among the caregivers just for comparison with ALS patients as regards the initial osteopathic evaluation.

\section{Interventions}

Patients were randomized (time point: T0) by a randomly generated list according to an initial single-blind design, followed by an open period (see below), in order to receive either OMT (n=7, OMT) or a standard-of-care treatment (physiotherapy twice a week, $\mathrm{n}=7, \mathrm{FKT}$ ). OMT was administered once a week for the first four weeks, and fortnightly for the following eight weeks. At the end of this period (T1), 10 patients received OMT (open period) once a week for the following eight weeks (T2) (Fig. 1).

SD were defined as impaired or altered functions of components of the somatic system including skeletal, arthrodial and myofascial structures, and their related vascular, lymphatic, and neural elements [6]. The osteopathic manoeuvres applied in the OMT group were performed by 5 -years experienced personnel and were individually tailored for each patient, according to Greenman's descriptions [12]. The OMT techniques were focused on the treatment of SD found 
during the re-evaluations performed before each session; structural (including myofascial release), visceral, and craniosacral techniques were performed as appropriate [4]. Treatment duration was always 40 minutes.

\section{Outcome Measures}

Primary: Adverse effects of treatment, if reported by patients or caregivers, were recorded by a medical doctor before starting each new session of OMT. A visual analogue scale (VAS) was included at T1 and T2 for those patients receiving OMT, focusing on the perceived satisfaction regarding the treatment.

Secondary: Each patient was evaluated in blind by a clinical neurologist at the three time points (T0, T1 and T2) for pain and QoL by using two dedicated questionnaires: the Brief Pain Inventory short form (BPI) and the McGill QoL (16 items). BPI allowed the calculation of the pain severity index (PSI) and the pain interference index (PII), averaging the respective items of the scale [13]. The McGill QoL allowed the calculation of: total score (TS), single-item summary score, macroareas scores: (a) physical or (b) psychological symptoms, (c) physical or (d) psychological well-being, and (e) support [14].

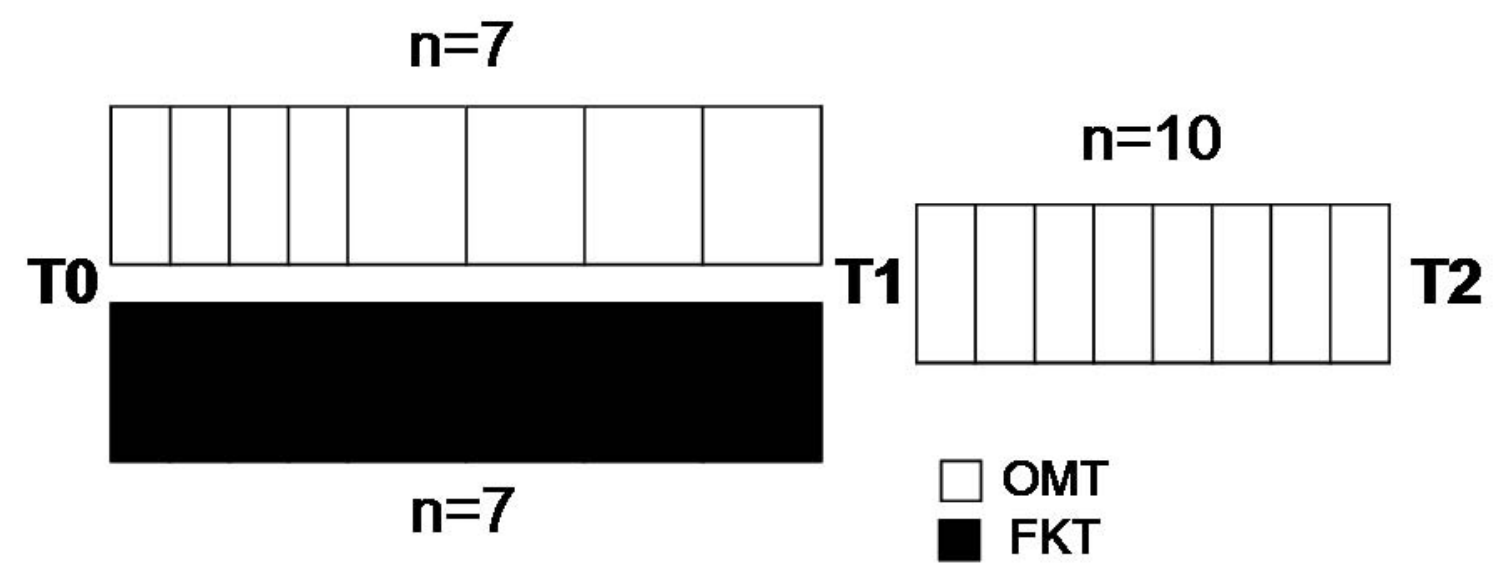

Fig. (1). Gantt chart of the study. During the first study period (T0-T1), 7 ALS patients received OMT once a week (small white blocks) for the first month and fortnightly during the following 8 weeks (big white blocks); during this period, 7 ALS patients were followed according to usual care (FKT arm, black bar). During the second study period (T1-T2), 10 ALS patients received OMT on a weekly basis.

Finally, two experienced Doctors of Osteopathic Medicine (D.O.) assessed all patients (and healthy controls), independently and blindly with respect to the initial treatment allocation, re-assessing them at T1 and T2. The observations of these two raters were not used for guiding treatments. The evaluation was performed according to a predecided structured scheme focusing on SD in order to check the same anatomical structures and qualify specific parameters (use of accessory muscles of breathing, direction of abdominal wall movement during inspiration, cervical or dorsal kyphosis, etc.); a careful rater training was also performed in advance. These evaluations were used for describing the effect of treatments on the initially found SD on a Goal Attainment Scale (GAS T0-T1 and GAS T1-T2) [15]. Briefly, GAS defines goals of treatment and describes their achievement on 5-point scale, in this case focusing on the number of identified SD: -2 (marked worsening, $\geq 50 \%$ ), -1 (mild worsening, $<50 \%$ ), 0 (unchanged), +1 (mild improvement, $<50 \%$ ), +2 (marked improvement, $\geq 50 \%$ ). We chose to set the 0 goal to "unchanged" because of the intrinsic progressing nature of ALS.

\section{Sample Size and Statistical Analysis}

Considering that patients with ALS might be especially fragile to manual therapies due to the important limb and trunk weakness and to the respiratory insufficiency when in recumbent position, obliging to a continuous and careful reassessment of the provided therapies, we decided that the presently reported sample size could be adequate for a preliminary protocol, able to generate preliminary data on which grounding future studies.

Data are shown as mean \pm standard deviation except where specifically noted. Statistical analysis was performed with GraphPad Prism, version 4.00 program. Either, unpaired sample, or repeated measures Student's $t$-test were used 
as appropriate and reported. Correlations were computed by the Pearson's $r$ test and differences between frequencies by the $\chi^{2}$ test. Significance was set at $p<0.05$.

\section{RESULTS}

\section{Osteopathic Characterization of ALS Patients}

Demographic and clinical characteristics of the recruited patients are listed in Table 1. OMT allocated patients had significantly faster DPI (more than two-fold), reflecting a tendency to more advanced diseases (lower scale scores) and shorter disease durations. Bulbar onset patients were 2 in the OMT group and 1 in the FKT. Only one OMT patient had PEG.

Table 1. Clinical and demographic data of the recruited patients at the different time points of the study.

\begin{tabular}{|l|c|c|c|}
\hline Time x Treatment & $\begin{array}{c}\text { T0 } \mathbf{x} \text { OMT } \\
\mathbf{n}=\mathbf{7}\end{array}$ & $\begin{array}{c}\text { T0 } \mathbf{x} \text { FKT } \\
\mathbf{n}=\mathbf{7}\end{array}$ & $\begin{array}{c}\text { T1 x OMT } \\
\mathbf{n}=\mathbf{1 0}\end{array}$ \\
\hline Age, yr & $54.0 \pm 11.6$ & $51.0 \pm 6.5$ & $50.3 \pm 7.9$ \\
\hline Sex, M/F & $5 / 2$ & $5 / 2$ & $8 / 2$ \\
\hline Onset, Spinal/Bulbar & $5 / 2$ & $6 / 1$ & $10 / 0$ \\
\hline Duration, mo & $17.1 \pm 4.5$ & $38.0 \pm 30.5$ & $(8-5.526 .2$ \\
& $(11-25)$ & $40.4 \pm 4.0$ & $33.9 \pm 9.9$ \\
\hline ALSFRS-R & $35.0 \pm 6.7$ & $(35-46)$ & $(16-46)$ \\
\hline DPI & $(28-46)$ & $0.32 \pm 0.23$ & $0.49 \pm 0.39$ \\
& $0.81 \pm 0.47 *$ & $(0.04-0.62)$ & $30.03-1.28)$ \\
\hline ALSSS & $(0.10-1.54)$ & $33.8 \pm 2.7$ & $(21-38)$ \\
\hline Norris & $29.5 \pm 5.3$ & $(31-39)$ & $74.9 \pm 19.7$ \\
& $(24-37)$ & $90.0 \pm 4.5$ & $(36-96)$ \\
\hline Riluzole, Y/N & $73.4 \pm 16.9$ & $(85-98)$ & $7 / 3$ \\
\hline
\end{tabular}

DPI, Disease Progression Index; FKT, usual care (physiotherapy); OMT, osteopathic manual treatment. Data are shown as mean \pm SD. ${ }^{*} p<0.05 v s$. FKT, two-tailed Student's $t$-test.

At study entry (T0), a cervico-dorsal SD emerged as particularly prevalent in ALS patients, being documented in 11 $(78 \%)$ of them and only in $4(28 \%)$ matched healthy controls $\left(\chi^{2} 7.04, p<0.01\right.$, OR 9.17, 95\% CI 1.27-79.25). No correlation was found with clinical characteristics (disease duration, ALSFRS-R score, ALS-SS, Norris score, DPI, prevalence of upper or lower motoneuron involvement), nor differences were found dichotomizing patients according to: site of onset (spinal vs. bulbar), riluzole use or El Escorial category (probable vs. definite) (data not shown).

Table 2. McGill QoL total score, summary and macroarea scores did not vary significantly between groups and over time. Mean \pm SD (range).

\begin{tabular}{|l|c|c|c|c|c|}
\hline & \multicolumn{2}{|c|}{ T0 } & \multicolumn{2}{|c|}{ T1 } & T2 \\
\hline Treatment & OMT & FKT & OMT & FKT & OMT \\
& $\mathbf{n = 7}$ & $\mathbf{n = 7}$ & $\mathbf{n}=7$ & $\mathbf{n}=\mathbf{1 0}$ \\
\hline Total score & $103.3 \pm 24.6$ & $103.3 \pm 25.4$ & $105.6 \pm 26.5$ & $106.0 \pm 18.1$ & $97.3 \pm 22.4$ \\
& $(72-138)$ & $(53-126)$ & $(69-140)$ & $(86-128)$ & $(64-135)$ \\
\hline Summary score & $7.7 \pm 1.9$ & $6.9 \pm 3.1$ & $6.1 \pm 2.6$ & $7.0 \pm 2.0$ & $6.9 \pm 1.9$ \\
& $(5-10)$ & $(0-10)$ & $(2-10)$ & $(5-9)$ & $(5-10)$ \\
\hline Physical symptoms & $19.7 \pm 6.4$ & $18.0 \pm 7.5$ & $21.1 \pm 5.8$ & $20.0 \pm 8.7$ & $20.8 \pm 7.7$ \\
& $(11-28)$ & $(5-29)$ & $(10-28)$ & $(4-30)$ & $(8-30)$ \\
\hline Psychological symptoms & $17.1 \pm 11.0$ & $15.1 \pm 8.4$ & $14.0 \pm 8.7$ & $13.7 \pm 5.3$ & $17.0 \pm 11.2$ \\
& $(3-31)$ & $(8-31)$ & $(3-28)$ & $(5-20)$ & $(2-34)$ \\
\hline Physical well-being & $7.6 \pm 2.6$ & $6.9 \pm 1.6$ & $6.0 \pm 3.2$ & $6.6 \pm 1.9$ & $7.1 \pm 1.7$ \\
& $(2-10)$ & $(4-8)$ & $(2-10)$ & $(4-9)$ & $(4-10)$ \\
\hline Psychological well-being & $48.4 \pm 9.2$ & $43.0 \pm 13.1$ & $47.4 \pm 10.9$ & $46.0 \pm 6.3$ & $45.0 \pm 7.6$ \\
& $(34-60)$ & $(15-53)$ & $(31-59)$ & $(38-55)$ & $(34-57)$ \\
\hline Support & $17.9 \pm 2.1$ & $16.6 \pm 2.8$ & $17.6 \pm 3.0$ & $17.0 \pm 3.3$ & $17.0 \pm 2.7$ \\
& $(15-20)$ & $(13-20)$ & $(14-20)$ & $(11-20)$ & $(12-20)$ \\
\hline
\end{tabular}




\section{Primary Aims: Feasibility, Tolerability and Satisfaction}

OMT was demonstrated feasible and safe over the whole study. All patients well adhered to treatment procedures. No significant side effects were reported. Only one patient reported moderately increased pain the day after the first treatment. New symptom onset was not recorded. Two OMT patients and two FKT ones dropped out at the end of T1 because of the impossibility of reaching treatment setting due to the advancing disease.

Overall OMT treated patients displayed high satisfaction with VAS scores of $8.34 \pm 0.46$ at T1 (n=7) and of $8.52 \pm$ 0.60 at T2 $(n=10) ; 5$ patients enrolled in the FKT group specifically asked for entering in the OMT arm during the T1$\mathrm{T} 2$ period.

\section{Secondary Aims: Pain, QoL and GAS}

8 patients at T0 (57\%) failed to report pain at the BPI, as well as 6 patients $(43 \%)$ at T1 and 7 patients $(50 \%)$ at T2. Regarding the period T0-T1, no BPI differences were found between the two time points and the two groups (mean delta PSI: -0.23 vs. 0.06, for OMT and FKT, respectively; mean delta PII: 0.71 vs. 1.48, for OMT and FKT, respectively). On the other hand, considering the following period (T1-T2, $\mathrm{n}=10)$, a tendency toward pain reduction over time was shown for PSI ( $p=0.05$, two-tailed paired Student's $t$-test; see Fig. 2), which did not reach statistical significance. In contrast, PII scores were unchanged ( $p=0.13$; Fig. 2).

McGill QoL TS, single item summary score and macroareas scores did not significantly change over time or between the two treatment groups (see Table 2).

Regarding blind osteopathic GAS scoring relative to the T0-T1 period, 3 patients $(43 \%)$ allocated to OMT and 2 (28\%) allocated to the FKT group showed a mild improvement (GAS score +1 ); none of the patients allocated to OMT and $2(28 \%)$ patients allocated to the FKT group worsened (GAS score -1$)\left(\chi^{2} p=0.12\right)$. Regarding the T1-T2 period, one patient (10\%) mildly improved, while 3 patients (30\%) mildly worsened in terms of number of SD; 6 patients $(60 \%)$ remained unchanged (GAS score 0; see Fig. 3).
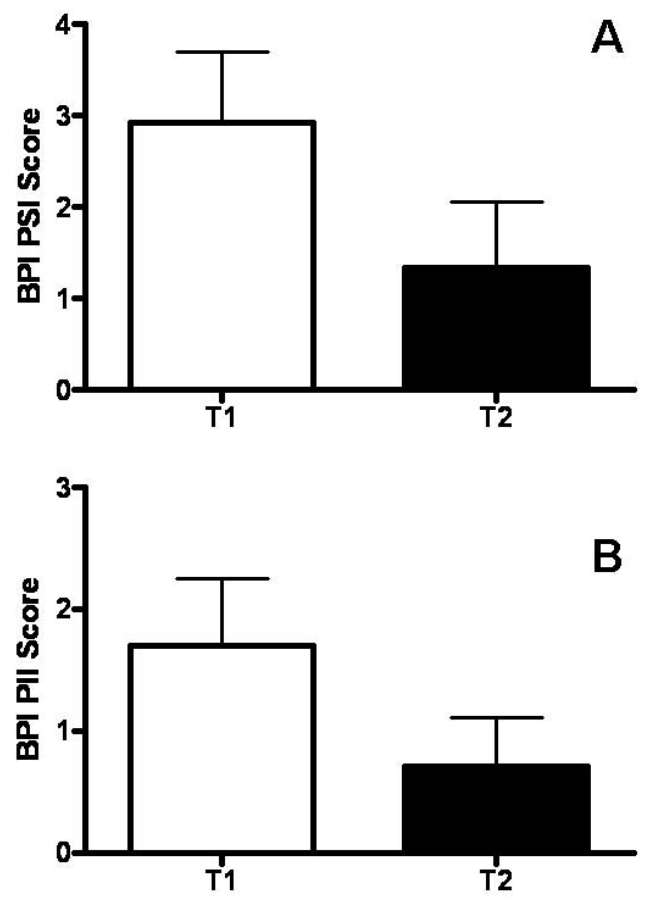

Fig. (2). (A) Pain severity showed a tendency to decrease over time in ALS patients between T1 and T2 (PSI, pain severity index of the BPI, $p=0.05$ ), while $(\mathbf{B})$ interference with daily life activities was unchanged (PII, $p=0.13$ ). Two-tailed paired Student's $t$-test; mean \pm SEM; $\mathrm{n}=10$. 


\section{DISCUSSION}

In this pilot study OMT procedures were clearly found feasible and safe in ALS patients. Furthermore, OMT was highly satisfactory for these patients. Nevertheless, no differences emerged in terms of secondary aims, i.e., the efficiency in modifying pain and QoL, both between the two treatment arms for the first study period and over time. Certainly, the limited sample size obliges to take these results cautiously since a tendency toward a reduction of the severity index was shown over the second study period (T1-T2). Notably, OMT-allocated patients were generally more severe and with shorter disease durations, underlying a significantly faster progression. This issue, unbalanced between the two groups of patients due to the limited sample size, might have been reasonably contributing to the obtained lack of significance and larger studies are needed to assess OMT effects on these selected outcomes.

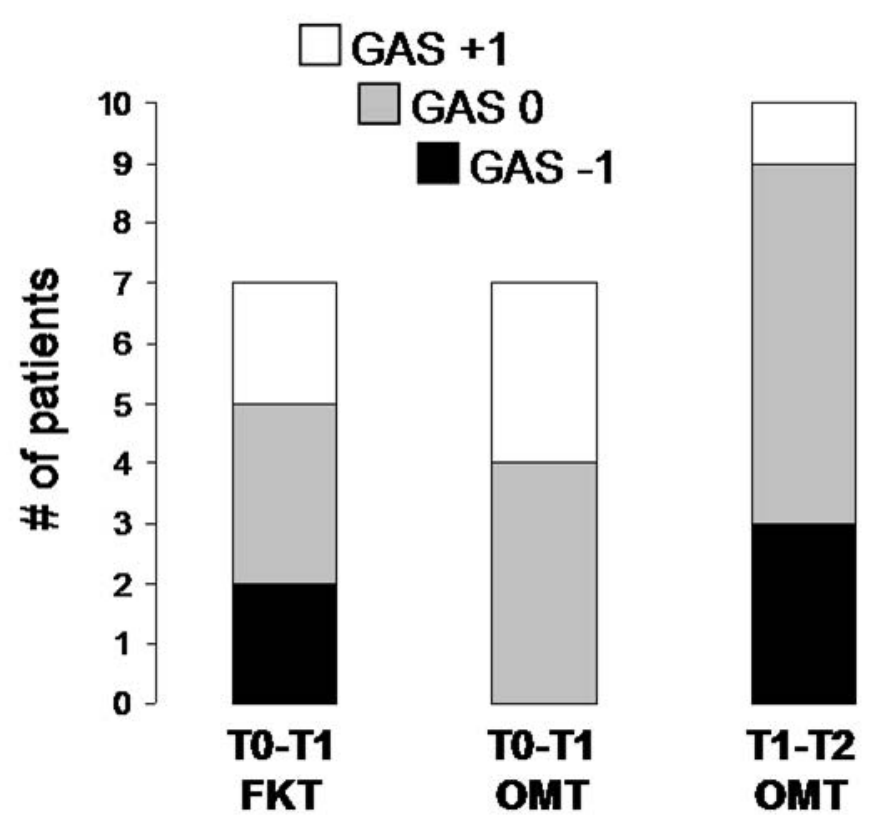

Fig. (3). Goal attainment scale (GAS) in ALS patients undergoing to OMT or FKT during the first period ( $\mathrm{n}=7$ for each arm, T0-T1; $\chi^{2} p=0.12$ ) and for those 10 patients completing the following two months (T1-T2 period).

Furthermore, BPI detects pain only over the last 24 hours, possibly not capturing the complexity of the relapsingremitting symptoms in a rapid progressing disease, such as ALS. Also, it is important to stress that several patients reported that pain was not a major problem if compared to the decreasing functional autonomy. In order to overcome this problem, previous attempts of detecting pain in ALS patients were done modifying the scale in order to refer to the last week [1]. Possibly, ALS-dedicated pain questionnaires could be developed and validated in the future in order to improve pain detection. Besides "hard" secondary outcomes, we decided to include a single-blind measure able to describe putative treatment-induced modifications in terms of osteopathic medicine. To our knowledge, this is the first attempt of blind scoring the achievement of specific osteopathic outcomes (in this case SD reduction) by using a patient-tailored scale. Goal attainment scaling was chosen, setting the desired expected outcome on the lack of osteopathic changes over time, considering the intrinsic worsening nature of ALS. Albeit no significant differences were reported between OMT and FKT arms, a tendency toward less worsening was shown in the OMT group, again suggesting that sample size could be increased before concluding. From our initial assessments, we conclude from this pilot study that an osteopathic substrate for manual treatment might be present in ALS patients, since they express more frequently a cervico-dorsal SD when compared to healthy matched controls. Albeit the significance of this dysfunction in terms of OMT is not exactly known, future studies may determine if this SD might respond to focused OMT approaches and if this result might affect pain, QoL or other outcomes, for example functionally-related. We regard this 
approach as fully justified by the lack of current effective treatments able to contrast disease-related pain and QoL impairment in ALS patients. In fact, the high level of satisfaction perceived by all those patients receiving OMT raises important questions related to what exactly is measured by the used outcomes. For example, we may postulate that the lack of QoL improvement we observed in front of elevated VAS scores indicates that specific areas of one patient's life might selectively benefit from OMT. Nevertheless, considering that OMT could be adapted to each phase of the disease and seems safe also in these fragile patients, we support the idea that could be an interesting option to explore in ALS. In fact, a multimodal treatment strategy might help in developing approaches patient-tailored able to ease sufferance for these individuals till the very end of their life, sustaining, at the same time, their caregivers.

\section{FUNDING}

Italian Academy of Osteopathic Medicine (AIMO).

\section{ETHICAL APPROVAL}

Protocol 129 10-JUN-2014; ethics committee of the University of Milano-Bicocca, Italy.

Raw data are available upon request from the Authors.

\section{CONFLICT OF INTEREST}

The authors confirm that this article content has no conflict of interest.

\section{ACKNOWLEDGEMENTS}

The Italian Association for ALS (AISLA; http:/www.aisla.it/) and the resting home "San Pietro"- Cooperative "La Meridiana" (Monza, Italy).

\section{REFERENCES}

[1] Chiò A, Canosa A, Gallo S, et al. Pain in amyotrophic lateral sclerosis: a population-based controlled study. Eur J Neurol 2012; 19(4): 551-5. [http://dx.doi.org/10.1111/j.1468-1331.2011.03540.x] [PMID: 21972798]

[2] Miller RG, Jackson CE, Kasarskis EJ, et al. Practice parameter update: the care of the patient with amyotrophic lateral sclerosis: multidisciplinary care, symptom management, and cognitive/behavioral impairment (an evidence-based review): report of the Quality Standards Subcommittee of the American Academy of Neurology. Neurology 2009; 73(15): 1227-33. [http://dx.doi.org/10.1212/WNL.0b013e3181bc01a4] [PMID: 19822873]

[3] Wells MR, Giantinoto S, D’Agate D, et al. Standard osteopathic manipulative treatment acutely improves gait performance in patients with Parkinson's disease. J Am Osteopath Assoc 1999; 99(2): 92-8. [http://dx.doi.org/10.7556/jaoa.1999.99.2.92] [PMID: 10079641]

[4] Rolle G, Tremolizzo L, Somalvico F, Ferrarese C, Bressan LC. Pilot trial of osteopathic manipulative therapy for patients with frequent episodic tension-type headache. J Am Osteopath Assoc 2014; 114(9): 678-85. [http://dx.doi.org/10.7556/jaoa.2014.136] [PMID: 25170037]

[5] Rivera-Martinez S, Wells MR, Capobianco JD. A retrospective study of cranial strain patterns in patients with idiopathic Parkinson's disease. J Am Osteopath Assoc 2002; 102(8): 417-22. [PMID: 12201545]

[6] Glossary of osteopathic terminology 2011. Available at: https://www.aacom.org/docs/default-source/insideome/got2011ed.pdf?sfvrsn=2

[7] Brooks BR, Miller RG, Swash M, Munsat TL. El Escorial revisited: revised criteria for the diagnosis of amyotrophic lateral sclerosis. Amyotroph Lateral Scler Other Motor Neuron Disord 2000; 1(5): 293-9. [http://dx.doi.org/10.1080/146608200300079536] [PMID: 11464847]

[8] Cedarbaum JM, Stambler N, Malta E, et al. The ALSFRS-R: a revised ALS functional rating scale that incorporates assessments of respiratory function. J Neurol Sci 1999; 169(1-2): 13-21. [http://dx.doi.org/10.1016/S0022-510X(99)00210-5] [PMID: 10540002]

[9] Hillel AD, Miller RM, Yorkston K, McDonald E, Norris FH, Konikow N. Amyotrophic lateral sclerosis severity scale. Neuroepidemiology 1989; 8(3): 142-50.

[http://dx.doi.org/10.1159/000110176] [PMID: 2725806]

[10] Norris FH Jr, Calanchini PR, Fallat RJ, Panchari S, Jewett B. The administration of guanidine in amyotrophic lateral sclerosis. Neurology 1974; 24(8): 721-8. [http://dx.doi.org/10.1212/WNL.24.8.721] [PMID: 4858705]

[11] Tremolizzo L, Ferrarese C, Appollonio I. Exploring limits of neuropsychological screening in ALS: the FAB problem. Amyotroph Lateral Scler Frontotemporal Degener 2013; 14(2): 157-8. [http://dx.doi.org/10.3109/21678421.2012.746990] [PMID: 23286758] 
[12] Greenman PE. Principles of Manual Medicine. $3^{\text {rd }}$ ed. Philadelphia, PA: Lippincott Williams \& Wilkins 2003.

[13] Bonezzi C, Nava A, Barbieri M, et al. Validazione della versione italiana del Brief Pain Inventory nei pazienti con dolore cronico. Minerva Anestesiol 2002; 68(7-8): 607-11. [PMID: 12244292]

[14] Beghi E, Pupillo E, Bonito V, et al. Randomized double-blind placebo-controlled trial of acetyl-L-carnitine for ALS. Amyotroph Lateral Scler Frontotemporal Degener 2013; 14(5-6): 397-405.

[http://dx.doi.org/10.3109/21678421.2013.764568] [PMID: 23421600]

[15] Kiresuk TJ, Sherman RE. Goal attainment scaling: A general method for evaluating comprehensive community mental health programs. Community Ment Health J 1968; 4(6): 443-53. [http://dx.doi.org/10.1007/BF01530764] [PMID: 24185570]

\section{(C) Maggiani et al.; Licensee Bentham Open}

This is an open access article licensed under the terms of the Creative Commons Attribution-Non-Commercial 4.0 International Public License (CC BY-NC 4.0) (https://creativecommons.org/licenses/by-nc/4.0/legalcode), which permits unrestricted, non-commercial use, distribution and reproduction in any medium, provided the work is properly cited. 